\title{
ON DECOMPOSITIONS OF NORMED UNITS IN ABELIAN GROUP RINGS
}

\author{
PETER DANCHEV \\ Department of Mathematics, Plovdiv State University, 4000 Plovdiv, \\ Bulgaria, pvdanchev@yahoo.com \\ Dedicated to the memory of William Davis Ullery \\ (March 10, 1954 - January 01, 2012)
}

\begin{abstract}
We strengthen some results of W. L. May (J. Algebra, 1976) by finding a criterion when a special decomposition of normed units in abelian group rings holds.

Key words: Groups, rings, group rings, units, decompositions, idempotents, nilpotents.
\end{abstract}

Abstrak. Pada paper ini akan diperkuat beberapa hasil dari W. L. May (J. Algebra, 1976) dengan menemukan sebuah kriteria kapan sebuah dekomposisi khusus dari unit bernorm dalam grup ring Abel dipenuhi.

Kata kunci: Group, ring, group ring, unit, dekomposisi, idempoten, nilpoten.

\section{Introduction}

Throughout this paper, let $R$ be a commutative unitary ring of arbitrary characteristic and let $G$ be an abelian multiplicative group. Besides, traditionally suppose $R G$ is the group ring of $G$ over $R$ with group of normed units (i.e., of augmentation 1) $V(R G)$. In fact, as usual, $R G$ is defined as the set $R G=$ $\left\{\sum_{g \in G} r_{g} g \mid r_{g} \in R\right\}$ with algebraic operations $\sum_{g \in G} r_{g} g+\sum_{g \in G} t_{g} g=\sum_{g \in G}\left(r_{g}+\right.$ $\left.t_{g}\right) g,\left(\sum_{g \in G} r_{g} g\right) \cdot\left(\sum_{h \in G} t_{h} h\right)=\sum_{g \in G} \sum_{h \in G} r_{g} \cdot t_{h} g \cdot h$ and $\sum_{g \in G} r_{g} g=\sum_{g \in G} t_{g} g$ $\Longleftrightarrow r_{g}=t_{g}$. Likewise, $U(R G)$ is the multiplicative group of $R G$, i.e. group of units in $R G$, and $V(R G)=\left\{\sum_{g \in G} r_{g} g \in U(R G) \mid \sum_{g \in G} r_{g}=1\right\}$.

Furthermore, let us assume that $N(R)$ is the nil-radical of $R$ and $G_{0}=\coprod_{p} G_{p}$ is the torsion subgroup of $G$ with $p$-component $G_{p}$. Also, let $i d(R)=\left\{e \in R: e^{2}=\right.$

2000 Mathematics Subject Classification: 20K21, 16U60, $16 \mathrm{~S} 34$

Received: 06-07-2010, revised: 27-12-2011, accepted: 27-01-2012. 
e\} be the set of all idempotents in $R, \operatorname{inv}(R)=\left\{p: p \cdot 1 \in R^{*}\right\}$, where $R^{*}$ is the unit group of $R, z d(R)=\{p: \exists r \in R \backslash\{0\}, p \cdot r=0\}$ and $\operatorname{supp}(G)=\left\{p: G_{p} \neq 1\right\}$. Following [5], we define the following three concepts:

$$
\begin{gathered}
I(N(R) G ; G)=\left\{\sum_{g \in G} r_{g} g\left(1-h_{g}\right) \mid r_{g} \in N(R), h_{g} \in G\right\}=N(R) \cdot I(R G ; G), \\
I(R G ; H)=\left\{\sum_{a \in G} f_{a} a\left(1-b_{a}\right) \mid f_{a} \in R, b_{a} \in H\right\},
\end{gathered}
$$

whenever $H \leq G$ and

$$
I d(R G)=\left\{\sum_{g \in G} e_{g} g \mid e_{g} \in i d(R), \sum_{g \in G} e_{g}=1, e_{g} \cdot e_{h}=0, g \neq h\right\} .
$$

It is a routine technical exercise to verify that $1+I(N(R) G ; G)$ meets $\operatorname{Id}(R G)$ only trivially and that $\operatorname{Id}(R G)=G$ if and only if $i d(R)=\{0,1\}$. All other unexplained explicitly notions and notations are standard and follow for the most part those from [5].

In 1976, Warren Lee May proved in [6] that if $\operatorname{supp}(G) \cap \operatorname{inv}(R)=\emptyset$ and $i d(R)=\{0,1\}$ (i.e., $R$ is indecomposable), then the following decomposition is valid:

$$
\text { (1) } V(R G)=G V\left(R G_{0}+N(R G)\right) \text {. }
$$

In [1] we extended this result by finding a necessary and sufficient condition proving that (1) holds if and only if either $G$ is torsion, or $G$ is torsion-free or mixed (i.e., in both cases it contains an element of infinite order) and no prime which is an order of an element of $G$ inverts in $R$.

Next, we obtained in [2] a criterion when the following decomposition is true:

$$
\text { (2) } V(R G)=G V\left(R G_{0}\right) \text {. }
$$

Clearly (1) and (2) are equivalent when $N(R G)=0$, i.e., by [6], when $N(R)=$ 0 and $\operatorname{supp}(G) \cap z d(R)=\emptyset$.

After this, we established in [4] a necessary and sufficient condition when the following more general decomposition is fulfilled:

$$
\text { (3) } V(R G)=I d(R G) V\left(R G_{0}\right) \text {, }
$$

provided $\operatorname{char}(R)$ is prime.

Evidently (2) and (3) are equivalent if $\operatorname{Id}(R G)=G$, i.e., if $i d(R)=\{0,1\}$. Notice the interesting fact from $[6]$ that $i d(R G)=\{0,1\}$ uniquely when $i d(R)=$ $\{0,1\}$ and $\operatorname{supp}(G) \cap i n v(R)=\emptyset$. 
The purpose of this short article is to generalize the aforementioned achievements by dropping off the restriction on the characteristic of the coefficient ring in (3) to be a prime integer and by considering the enlarged decomposition

$$
\text { (4) } V(R G)=I d(R G) V\left(R G_{0}+N(R G)\right) \text {. }
$$

The motivation for making this is that the decomposition (4) is rather important for application on description of the structure of $V(R G)$ (see, e.g., [5] and $[6])$. In order to do that, we will refine the technique used in [6] and [3].

\section{Main Results}

Before stating and proving our chief assertion, we need one more technicality from [3], stated below as Proposition 2.1. First, some preliminaries:

Suppose $\phi: G \rightarrow G / G_{0}$ is the natural map which is, actually, a surjective homomorphism. It is well known that it can be linearly extended in the usual way $\Phi\left(\sum_{g \in G} r_{g} g\right)=\sum_{g \in G} r_{g} \phi(g)=\sum_{g \in G} r_{g} g G_{0}$ to the epimorphism $\Phi: R G \rightarrow$ $R\left(G / G_{0}\right)$ of $R$-group algebras with kernel $I\left(R G ; G_{0}\right)$. Its restriction on $V(R G)$ gives a homomorphism $\Phi_{V(R G)}: V(R G) \rightarrow V\left(R\left(G / G_{0}\right)\right)$ with kernel $\left(1+I\left(R G ; G_{0}\right)\right) \cap$ $V(R G)$ while it is self-evident that $\Phi_{I d(R G)}: \operatorname{Id}(R G) \rightarrow \operatorname{Id}\left(R\left(G / G_{0}\right)\right)$ is a surjective homomorphism (= epimorphism) with kernel $\operatorname{Id}\left(R G_{0}\right)$.

Let $P$ be a commutative unitary ring with $|i d(P)|>2$ and let $P=R_{1} \times$ $\cdots \times R_{n}$ where each $R_{i}$ is an indecomposable subring of $P$ for $i \in[1, n]$. It is straightforward to see that $\operatorname{inv}(P) \subseteq \operatorname{inv}\left(R_{i}\right)$ for every index $1 \leq i \leq n$, while the converse inclusion may not be ever fulfilled - see the example listed below in Remark 2.

Moreover, if $\operatorname{supp}(G) \cap i n v(K)=\emptyset$ for every indecomposable subring $K$ of $R$, then $\operatorname{supp}(G) \cap i n v(F)=\emptyset$ for each finitely generated subring $F$ of $R$, and hence it is elementary to see that $\operatorname{supp}(G) \cap i n v(R)=\emptyset$ as well. However, the converse does not hold.

Observe also that $\operatorname{inv}(\{0\})=\emptyset$.

Proposition 2.1. Let $G$ be a group and $R$ a ring such that $\operatorname{supp}(G) \cap \operatorname{inv}(K)=\emptyset$ for any indecomposable subring $K$ of $R$. Then

$$
\left(1+I\left(R G ; G_{0}\right)\right) \cap V(R G) \subseteq V\left(R G_{0}+N(R G)\right) .
$$

Remark 1. The above supersedes ([6], Proposition 4) provided that $R$ is indecomposable. Besides, in the original formulation of ([3], Proposition 3 ) there is a misprint, namely there $i n v(R)$ should be written and read as $i n v(K)$ for each indecomposable subring $K$ of $R$. In this way, Proposition 2.1 formulated above is the correct statement. 
So, we have all the ingredients to prove the following assertion that is our major tool which, as aforementioned, improves the corresponding claim from [1].

Theorem 2.2. Suppose $R$ is a ring and $G$ is a group. Then

$$
V(R G)=\operatorname{Id}(R G) V\left(R G_{0}+N(R G)\right)
$$

if and only if

(a) $G=G_{0}$, or

(b) $G \neq G_{0}$ and $\operatorname{supp}(G) \cap i n v(K)=\emptyset$ for all indecomposable subrings $K$ of $R$.

ProOF. " $\Rightarrow "$. If $G$ is torsion, the equality holds no matter what $R$ is. So we will assume that there exists $g \in G \backslash G_{0}$, whence $g^{n} \neq 1$ for every $n \in \mathbb{N}$. We will show below that $i d\left(R G_{0}\right) \backslash i d(R)=\emptyset$ whenever $\operatorname{supp}(G) \cap i n v(K) \neq \emptyset$ for each indecomposable subring $K$ of $R$, which is impossible. Letting $e \in i d\left(R G_{0}\right)$, we have $e \in i d\left(F G_{0}\right)$ for some finitely generated subring $F$ of $R$, whence there exists a finite number of indecomposable subrings $K_{1}, \ldots, K_{t}$ such that $F=K_{1} \times \cdots \times K_{t}$. That is why, without loss of generality, we may further assume that $R$ is finitely generated itself.

In fact, let $e \in i d\left(K G_{0}\right)$ for some arbitrary but a fixed indecomposable subring $K$ with $e \notin i d(R)$. It is long known that $e$ can be represented like this: $e=$ $\frac{1}{n}\left(1+b+\cdots+b^{n-1}\right)$ where $n \in \operatorname{supp}(G) \cap i n v(K)$ and $1 \in K$, whereas $b \in G$ with $\operatorname{order}(b)=n$. It is obvious that $e g+(1-e) \in V(K G)$ with the inverse $e g^{-1}+(1-e)$. Thus we may write $e g+(1-e)=h(b+c)$ where $h=e_{1} g_{1}+\cdots+e_{s} g_{s} \in \operatorname{Id}(R G)$, $b \in R G_{0}$ and $c \in N(R G)$. It is readily seen that this equality can be written as follows:

$$
e\left(g h^{-1}-b\right)+(1-e)\left(h^{-1}-b\right)=c .
$$

Since $e(1-e)=0$ and there is some $m \in \mathbb{N}$ with the property $c^{m}=0$, we obtain that

$$
e\left(g h^{-1}-b\right)^{m}+(1-e)\left(h^{-1}-b\right)^{m}=0 .
$$

Multiplying both sides with $e$ and $1-e$, respectively, the last reduces to the equalities

$$
e\left(g h^{-1}-b\right)^{m}=0=(1-e)\left(h^{-1}-b\right)^{m} .
$$

Apparently, either $g h^{-1}$ or $h^{-1}$ is torsion-free. Suppose by symmetry $h^{-1}=$ $e_{1} g^{-1}+\cdots+e_{s} g_{s}^{-1}$ is torsion-free. Therefore, there exists an index $j \in[1, s]$ such that $e_{j} \neq 0$ and $g_{j}^{-1}$ is torsion-free. Since both $b \in R G_{0}$ and $1-e=\sum_{d \in G_{0}} r_{d} d \in$ 
$R G_{0}$ (with $r_{d} \in R$ ), one may observe that in view of the Newton's binomial formula $(1-e)\left(h^{-1}-b\right)^{m}=0$ can be written as

$$
\sum_{d \in G_{0}} r_{d} d h^{-m}+\sum_{t \in G_{0}} \sum_{0 \leq i \leq m-1} f_{t} t h^{-i}=0
$$

for $f_{t} \in R$. Evidently, $h^{-m}=e_{1} g^{-m}+\cdots+e_{s} g_{s}^{-m}$ and $h^{-i}=e_{1} g^{-i}+\cdots+e_{s} g_{s}^{-i}$ with $e_{j} h^{-m}=e_{j} g_{j}^{-m}$ and $e_{j} h^{-i}=e_{j} g_{j}^{-i}$. Furthermore, multiplying both sides of the above sum's equality with $e_{j}$, we deduce that

$$
\sum_{d \in G_{0}} e_{j} r_{d} d g_{j}^{-m}+\sum_{t \in G_{0}} \sum_{0 \leq i \leq m-1} e_{j} f_{t} t g_{j}^{-i}=0
$$

It is clear that the last sum is now in canonical form where the two members in the left hand-side and in the right hand-side of the sign " + are disjoint as well. That is why $e_{j} r_{d}=0$ for each $d \in G_{0}$ and thus $e_{j}(1-e)=0$, i.e., $e_{j}=e_{j} e$. However, as written above, $e=n^{-1}\left(1+b+\cdots+b^{n-1}\right)$ and hence $1-e=1-n^{-1}-n^{-1} b-$ $\cdots-n^{-1} b^{n-1}$. It follows now that $r_{1}=1-n^{-1}$ and $r_{d}=-n^{-1}$ for $d \neq 1$, whence $e_{j}\left(1-n^{-1}\right)=0$ and $e_{j} n^{-1}=0$ which assures that $e_{j}=0$, a contradiction. This substantiates our claim that $i d\left(R G_{0}\right) \backslash i d(R)=\emptyset$, that is, $\operatorname{supp}(G) \cap i n v(K)=\emptyset$ as stated.

$" \Leftarrow "$. Suppose $\Phi$ is the map defined as in lines before Proposition 2.1. It is clear that $\Phi(V(R G)) \subseteq V\left(R\left(G / G_{0}\right)\right)$. Moreover, [5] allows us to write that

$$
V\left(R\left(G / G_{0}\right)\right)=I d\left(R\left(G / G_{0}\right)\right) \times\left(1+I\left(N(R)\left(G / G_{0}\right) ; G / G_{0}\right)\right) .
$$

As observed above, $\Phi(\operatorname{Id}(R G))=\operatorname{Id}\left(R\left(G / G_{0}\right)\right)$ and, moreover, it is easy to check that $\Phi(1+I(N(R) G ; G))=1+I\left(N(R)\left(G / G_{0}\right) ; G / G_{0}\right)$. Furthermore, one sees that $\Phi(V(R G)) \subseteq \Phi(I d(R G)) \Phi(1+I(N(R) G ; G))=\Phi(I d(R G)(1+I(N(R) G ; G)))=$ $\Phi(I d(R G) \times(1+I(N(R) G ; G)))$. But since $I d(R G) \times(1+I(N(R) G ; G)) \subseteq V(R G)$, the above inclusion is tantamount to

$$
\Phi(V(R G))=\Phi(I d(R G) \times(1+I(N(R) G ; G)))
$$

Observe that $1+I(N(R) G ; G) \subseteq 1+N(R) G \subseteq 1+N(R G) \subseteq V\left(R G_{0}+\right.$ $N(R G)) \times R^{*}$, so that $1+I(N(R) G ; G) \subseteq V\left(R G_{0}+N(R G)\right)$ - see also Proposition 2.3 listed below. Thus, applying Proposition 2.1, $\operatorname{ker} \Phi \subseteq V\left(R G_{0}+N(R G)\right)$ and it follows that $V(R G)=I d(R G) V\left(R G_{0}+N(R G)\right)$ as expected.

Remark 2. The next example illustrates that both Proposition 2.1 and Theorem 2.2 are not longer true if $R$ fails to have the required property that for any its indecomposable subring $K$ the intersection $\operatorname{supp}(G) \cap i n v(K)$ is empty. In other words, $\operatorname{supp}(G) \cap \operatorname{inv}(K)=\emptyset$ cannot be changed to $\operatorname{supp}(G) \cap i n v(R)=\emptyset$. 
Given $R=\mathbb{Z}_{2} \times \mathbb{Z}_{3}$ and take $G=\langle g, t\rangle$ where $o(g)=\infty$ and $o(t)=2$. Then $G \neq G_{0}$ and $\operatorname{inv}(R)=\mathbb{P} \backslash\{2,3\} ;$ besides $\operatorname{inv}\left(\mathbb{Z}_{2}\right)=\mathbb{P} \backslash\{2\}$ and $\operatorname{inv}\left(\mathbb{Z}_{3}\right)=\mathbb{P} \backslash\{3\}$ so that $\operatorname{inv}(R)=\operatorname{inv}\left(\mathbb{Z}_{2}\right) \cap \operatorname{inv}\left(\mathbb{Z}_{3}\right)$ and $\operatorname{inv}(R) \subset \operatorname{inv}\left(\mathbb{Z}_{2}\right), \operatorname{inv}(R) \subset \operatorname{inv}\left(\mathbb{Z}_{3}\right)$. Observe also that $\operatorname{char}(R)=6$ and $z d(R)=\{2,3\}$. We further have $R G=\mathbb{Z}_{2} G \times$ $\mathbb{Z}_{3} G, I d(R G)=G \times G, R G_{0}=\mathbb{Z}_{2} G_{0} \times \mathbb{Z}_{3} G_{0}$, and $N(R G)=N\left(\mathbb{Z}_{2} G\right) \times\{0\}$. Clearly, $V\left(R G_{0}+N(R G)\right) \subseteq \mathbb{Z}_{2} G \times \mathbb{Z}_{3} G_{0}$. Thus $I d(R G) V\left(R G_{0}+N(R G)\right) \subseteq \mathbb{Z}_{2} G \times G \cdot \mathbb{Z}_{3} G_{0}$. It is a routine technical exercise to verify that $e=2+2 t=\frac{1}{2}(4+4 t)=\frac{1}{2}(1+t)$ is an idempotent in $\mathbb{Z}_{3} G$, because $\operatorname{supp}(G) \cap i n v\left(\mathbb{Z}_{3}\right) \neq \emptyset$. Define $v \in V(R G)$ by $v=(1, e+g(1-e))$, the inverse $v^{-1}$ being obtained by replacing $g$ with $g^{-1}$. We calculate $v=(1,2+2 t+2 g+g t)$, hence $v \notin \mathbb{Z}_{2} G \times G \cdot \mathbb{Z}_{3} G_{0}$, and consequently $v \notin \operatorname{Id}(R G) V\left(R G_{0}+N(R G)\right)$ as expected. Therefore Theorem 2.2 will be wrong if only $\operatorname{supp}(G) \cap \operatorname{inv}(R)=\emptyset$ is required.

Moreover, if assuming just that $\operatorname{supp}(G) \cap \operatorname{inv}(R)=\emptyset$ is satisfied, then $v$ chosen as above will work again to provide a counterexample to Proposition 2.1. In fact, $v=(1,1)+(0,1+2 t+2 g+g t)$ with $(0,1+2 t+2 g+g t) \in I\left(R G ; G_{0}\right)$, whence $v \in V(R G) \cap\left(1+I\left(R G ; G_{0}\right)\right)$, as wanted. The example is shown.

We will demonstrate now one more useful relation.

Proposition 2.3. Suppose $R$ is a ring and $G$ is a group. Then the following decomposition holds:

$$
V\left(R G_{0}+N(R G)\right)=V\left(R G_{0}\right)(1+I(N(R G) ; G)) .
$$

Proof. Clearly, the left hand-side contains the right hand-side.

As for the converse implication, choose $v \in V\left(R G_{0}+N(R G)\right)$ hence $v=b+c$ where $b \in R G_{0}$ and $c \in N(R G)$. Since $b+c \in V(R G)$ and a unit plus a nilpotent is again a unit (note that this is true only in commutative rings), we have that $b \in U\left(R G_{0}\right)$. Even more, we may take $b \in V\left(R G_{0}\right)$ by adding the nilpotent $\pm a=\operatorname{aug}(c) \in N(R)$. So, $c$ can be taken to lie in $I(N(R G) ; G)=N(R G) I(R G ; G)$. In more precise words, $v=b+c=b+a+c-a=b^{\prime}+c^{\prime} \in V\left(R G_{0}\right)+I(N(R G) ; G)$ where $b^{\prime}=b+a \in V\left(R G_{0}\right)$ and $c^{\prime}=c-a \in I(N(R G) ; G)$. Furthermore, $v=$ $b\left(1+b^{-1} c\right) \in V\left(R G_{0}\right)(1+I(N(R G) ; G))$ as required.

So, Theorem 2.2 can be reformulated like this:

Theorem 2.2'. Suppose $R$ is a ring and $G$ is a group. Then

$$
V(R G)=I d(R G) V\left(R G_{0}\right)(1+I(N(R G) ; G))
$$

if and only if

(i) $G=G_{0}$ or

(ii) $G \neq G_{0}$ and $\operatorname{supp}(G) \cap i n v(K)=\emptyset$ for every indecomposable subring $K$ of $R$. 
Note that it can be shown that $V(R G)=\operatorname{Id}(R G) V\left(R G_{0}\right)(1+I(N(R G) ; G))$ implies $V(K G)=\operatorname{Id}(K G) V\left(K G_{0}\right)(1+I(N(K G) ; G))$ for all all indecomposable subrings $K$ of $R$.

As direct consequences, we derive the following affirmations.

Corollary 2.4. ([1]) Suppose $R$ is a ring and $G$ is a group. Then $V(R G)=$ $G V\left(R G_{0}+N(R G)\right) \Longleftrightarrow$ (a) $G=G_{0}$ or (b) $G \neq G_{0}$, id(R)=\{0,1\} and $\operatorname{supp}(G) \cap \operatorname{inv}(R)=\emptyset$.

Proof. Observe that $G V\left(R G_{0}+N(R G)\right) \subseteq I d(R G) V\left(R G_{0}+N(R G)\right) \subseteq V(R G)$. In virtue of Theorem 2.2 one needs to illustrate that $R$ is indecomposable. If $r \in i d(R)$, then $r g+(1-r) \in V(R G)$ for some $g \in G \backslash G_{0}$. Hence $r g+1-r=a(b+c)$ for some $a \in G, b \in R G_{0}$ and $c \in N(R G)$. As above, $b \in V\left(R G_{0}\right)$ and $r g a^{-1}+(1-$ $r) a^{-1}=b+c$, so that $r g a^{-1}+(1-r) a^{-1}-b=r\left(g a^{-1}-b\right)+(1-r)\left(a^{-1}-b\right)=c$. Furthermore, again as we previously observed, $r\left(g a^{-1}-b\right)^{m}=0=(1-r)\left(a^{-1}-b\right)^{m}$. However, $g a^{-1}$ and $a^{-1}$ cannot be torsion together, so that one of them is torsionfree; assume by symmetry that so is $g a^{-1}=h$. Thus $r(h-b)^{m}=0$ can be written in accordance with the Newton's binomial formula as $r\left(\sum_{t \in G_{0}} \sum_{0<i<m} f_{t} t h^{i}\right)=0$ for some $f_{t} \in R$ such that the ring coefficient $f_{t}$ stated before $h^{m}$ is exactly 1 . Moreover, the sum is obviously in canonical record. This immediately forces that $r=0$; the other possibility ensures that $1-r=0$, i.e., $r=1$ as desired.

The following strengthens the listed above equality (3) from [4].

Corollary 2.5. Let $R$ be a ring and let $G$ be a group. Then $V(R G)=\operatorname{Id}(R G) V\left(R G_{0}\right)$ if and only if

(a) $G=G_{0}$, or

(b) $G \neq G_{0}, N(R)=0$ and $\operatorname{supp}(G) \cap(i n v(K) \cup z d(R))=\emptyset$ for each indecomposable subring $K$ of $R$.

Proof. Because $\operatorname{Id}(R G) V\left(R G_{0}\right) \subseteq I d(R G) V\left(R G_{0}+N(R G)\right) \subseteq V(R G)$, what suffices to demonstrate is that $N(R G)=0$, which in the sense of [6] is precisely $N(R)=0$ and $\operatorname{supp}(G) \cap z d(R)=\emptyset$. Certainly, this is also tantamount to $N\left(R G_{0}\right)=0$ since $\operatorname{supp}(G)=\operatorname{supp}\left(G_{0}\right)$.

And so, choose $0 \neq z=f_{1} b_{1}+\cdots+f_{s} b_{s} \in N\left(R G_{0}\right)$ with $f_{i} \neq 0$ for any $i \in[1, s]$, whence $y=1+z(1-g) \in V(R G)$ whenever $g \in G \backslash G_{0}$. Thus we may write $1+z-z g=u v$ where $u=e_{1} g_{1}+\cdots+e_{s} g_{s} \in \operatorname{Id}(R G)$ with $e_{1}, \cdots, e_{s} \neq 0$ and $v=r_{1} c_{1}+\cdots+r_{s} c_{s} \in V\left(R G_{0}\right)$. Furthermore, one can write that

$(*)$

$1+f_{1} b_{1}+\cdots+f_{s} b_{s}-f_{1} b_{1} g-\cdots-f_{s} b_{s} g=\left(e_{1} g_{1}+\cdots+e_{s} g_{s}\right)\left(r_{1} c_{1}+\cdots+r_{s} c_{s}\right)$. 
Observe that there is an index $j \in[1, s]$ such that $e_{j} f_{1} \neq 0$; otherwise $0=$ $e_{1} f_{1}+\cdots+e_{s} f_{1}=\left(e_{1}+\cdots+e_{s}\right) f_{1}=f_{1}=0$, a contradiction. Thus, multiplying both sides of the above equality $(*)$ with $e_{j}$, we deduce that

$(* *)$

$$
e_{j}+e_{j} f_{1} b_{1}+\cdots+e_{j} f_{s} b_{s}-e_{j} f_{1} b_{1} g-\cdots-e_{j} f_{s} b_{s} g=e_{j} r_{1} g_{j} c_{1}+\cdots+e_{j} r_{s} g_{j} c_{s} .
$$

However, even if $b_{1}=1$, the situation $e_{j} f_{2}=\cdots=e_{j} f_{s}=0$ with $e_{j}+e_{j} f_{1}=0$ is impossible because it will lead to $e_{j}\left(f_{1} b_{1}+\cdots+f_{s} b_{s}\right)=-e_{j} b_{1} \in N\left(R G_{0}\right)$. Thus there exists $m \in \mathbb{N}$ with $e_{j} b_{1}^{m}=0$. But this implies that $e_{j}=0$ which is false. Furthermore, since the equality (**) is in canonical form, we derive that $g_{j} \in G_{0}$ and hence $g \in G_{0}$, contrary to our choice. Finally, this gives that $N\left(R G_{0}\right)=0$, i.e., $N(R G)=0$ as claimed.

Remark 3. When $\operatorname{char}(R)$ is a prime, say $p$, in [4] we obtained the conditions $G=G_{0}$ or $G \neq G_{0}=1$ and $N(R)=0$ which are obviously equivalent to these presented above. In fact, this is so since $\operatorname{char}(R)=p$ insures that $z d(R)=\{p\}$ and hence $\operatorname{supp}(G) \cap z d(R)=\emptyset$ because $i n v(R)$ contains all primes but $p$ and thus $\operatorname{supp}(G) \cap \operatorname{inv}(R)=\emptyset$ holds only when $\operatorname{supp}(G)=\emptyset$, i.e., when $G_{0}=1$.

Corollary 2.6. ([2]) Let $R$ be a ring and $G$ a group. Then $V(R G)=G V\left(R G_{0}\right)$ $\Longleftrightarrow \quad$ (i) $G=G_{0}$ or (ii) $G \neq G_{0}, i d(R)=\{0,1\}, N(R)=0$ and $\operatorname{supp}(G) \cap$ $(\operatorname{inv}(R) \cup z d(R))=\emptyset$.

Proof. Observe that $G V\left(R G_{0}\right) \subseteq I d(R G) V\left(R G_{0}\right) \subseteq V(R G)$. So, in view of Corollary 2.5 what we need to show is that $\operatorname{Id}(R G)=\bar{G}$ or, equivalently, $i d(R)=$ $\{0,1\}$, provided $V(R G)=G V\left(R G_{0}\right)$ is valid.

In fact, $I d(R G) \subseteq V(R G)$, whence $\operatorname{Id}(R G) \subseteq G V\left(R G_{0}\right)$ and so $\operatorname{Id}(R G)=$ $I d(R G) \cap\left(G V\left(R G_{0}\right)\right)$. Referring to the modular law, it is not difficult to see that the last intersection equals to $G\left(\operatorname{Id}(R G) \cap V\left(R G_{0}\right)\right)=G I d\left(R G_{0}\right)$. Consequently, $I d(R G)=G I d\left(R G_{0}\right)$. Suppose now that there exists $r \in i d(R) \backslash\{0,1\}$. Then $r g+(1-r) \in I d(R G)$ whenever $g \in G \backslash G_{0}$. Thus $r g+1-r=a\left(f_{1} b_{1}+\cdots+f_{s} b_{s}\right)=$ $f_{1} a b_{1}+\cdots+f_{s} a b_{s}$, where $a \in G$ and $f_{1} b_{1}+\cdots+f_{s} b_{s} \in \operatorname{Id}\left(R G_{0}\right)$. Since both sides are in canonical record, one may have $a \in G_{0}$ and hence $g \in G_{0}$ which is wrong. That is why $i d(R)$ contains only two elements as asserted.

Remark 4. In ([1], p. 157, line 18) the expression " $h \in H$ " should be written and read as " $h \in G "$.

We finish off with the following question of interest.

Problem 1. Under the given above limitations on $R$ and $G$, find a necessary and sufficient condition only in terms associated with $R$ and $G$ when the decomposition 


$$
V(R G)=I d(R G) V\left(R G_{0}+N(R) G\right)
$$

is fulfilled.

Acknowledgement The author would like to thank the anonymous referee for his/her careful reading of the manuscript.

\section{References}

[1] Danchev, P.V., "A note on a formula of May concerning units in abelian group rings", Bull. Allahabad Math. Soc. 23(1) (2008), 155 - 158.

[2] Danchev, P.V., "Note on a decomposition of normalized unit groups in abelian group algebras", Bull. Allahabad Math. Soc. 23(2) (2008), 365 - 368.

[3] Danchev, P.V., "Idempotent units of commutative group rings", Commun. Algebra 38(12) (2010), 4649 - 4654.

[4] Danchev, P.V., "On some idempotent-torsion decompositions of normed units in commutative group rings", J. Calcutta Math. Soc. 6(1) (2010), 31 - 34

[5] Karpilovsky, G., "Units of commutative group algebras", Expo. Math. 8 (1990), 247 - 287.

[6] May, W.L., "Group algebras over finitely generated rings", J. Algebra 39(4) (1976), 483 511. 